\title{
Strain Driven Phase Decomposition in Ion-Beam Sputtered $\mathrm{Pr}_{1-X} \mathrm{Ca}_{X} \mathrm{MnO}_{3}$ Films
}

\author{
Benedikt Ifland, Joerg Hoffmann, Thilo Kramer, Malte Scherff, \\ Stephanie Mildner, and Christian Jooss
}

\begin{abstract}
Institute of Materials Physics, University of Goettingen, Friedrich-Hund-Platz 1, 37077 Goettingen, Germany
Correspondence should be addressed to Christian Jooss; jooss@material.physik.uni-goettingen.de
\end{abstract}

Received 12 June 2015; Accepted 29 July 2015

Academic Editor: Ying-Hao Chu

Copyright (c) 2015 Benedikt Ifland et al. This is an open access article distributed under the Creative Commons Attribution License, which permits unrestricted use, distribution, and reproduction in any medium, provided the original work is properly cited.

\begin{abstract}
The deposition of heteroepitaxial thin films on single crystalline substrates by means of physical deposition methods is commonly accompanied by mechanical strain due to lattice mismatch and defect generation. Here we present a detailed analysis of the influence of strain on the Mn solubility of $\mathrm{Pr}_{1-X} \mathrm{Ca}_{X} \mathrm{MnO}_{3}$ thin films prepared by ion-beam sputtering. Combining results from X-ray diffraction, transmission electron microscopy and in situ hot-stage stress measurements, we give strong evidence that large tensile strain during deposition limits the Mn solubility range of the Perovskite phase to near-stoichiometric composition. Mn excess gives rise to $\mathrm{MnO}_{z}$ precipitates and the precipitation seems to represent a stress relaxation path. With respect to size and density of the precipitates, the relaxation process can be affected by the choice of substrate and the deposition parameters, that is, the deposition temperature and the used sputter gas.
\end{abstract}

\section{Introduction}

$\mathrm{Pr}_{1-X} \mathrm{Ca}_{X} \mathrm{MnO}_{3}$ (PCMO) belongs to the class of Perovskitetype oxides with the general formula $\mathrm{ABO}_{3}$ which exhibit rich and fascinating electronic and magnetic properties. These properties can be tailored via doping. In the case of the holedoped PCMO, the replacement of rare-earth element Pr on the $\mathrm{A}$-sites by the alkaline-earth element $\mathrm{Ca}$ gives rise to a series of different electronic and magnetic ground states. Above $X=0.3$, charge ordered phases are observed which show colossal resistance effects in external fields such as the colossal magnetoresistance (CMR) [1].

These remarkable properties are sensitively related to the subtle balance between the interactions of spin, lattice, charge, and orbital degrees of freedom which can dramatically change due to defects and strain. Small changes in bonding angles or bonding lengths of the octahedral tilt system can significantly alter the transfer integral [2] and therefore the magnetic exchange interactions and charge carrier mobility [3]. Consequently, without taking into account lattice disorder and stress-strain state, the understanding of structure-property relations of manganite thin films is hardly possible.

Strain appears in heteroepitaxially grown thin films due to the lattice mismatch (difference in lattice constants) and the thermal mismatch (difference in thermal expansion coefficients) between the substrate and the film. The effect of strain on charge ordering and the CMR in PCMO have been indeed observed in many studies [4-6].

In addition to lattice and thermal mismatch, the strain state of thin films is influenced by preparation induced defects. Sputter deposition of oxide films is commonly accompanied by the formation and annihilation of point-like intrinsic defects (e.g., Schottky defects) or nonstoichiometric defects (e.g., oxygen vacancies). Such defects can change the global lattice constant and can induce local disorder in the octahedral tilt system. Nonstoichiometric defects can also change the doping level. The role of electron-doping oxygen vacancies and A-site as well as B-site cation deficiencies in transport and magnetic properties has been, for example, studied in $[7,8]$. 


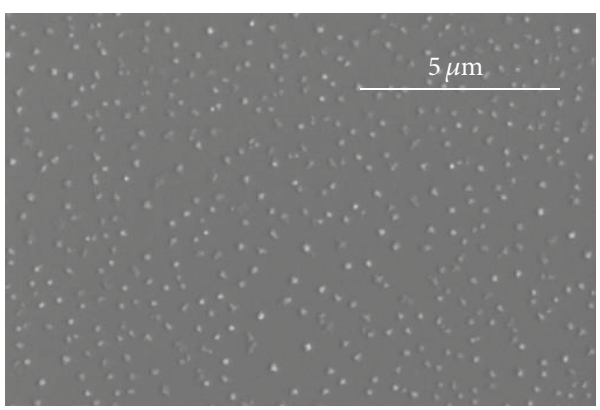

(a)

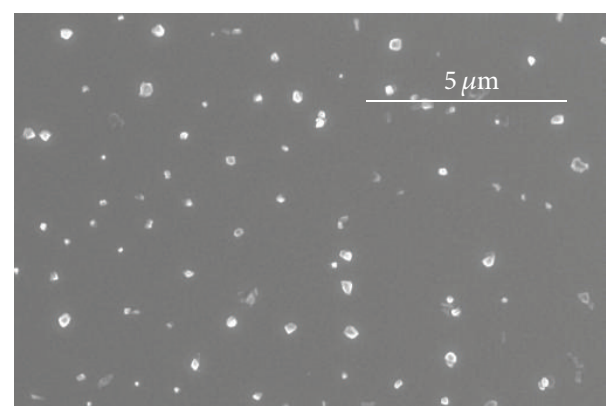

(b)

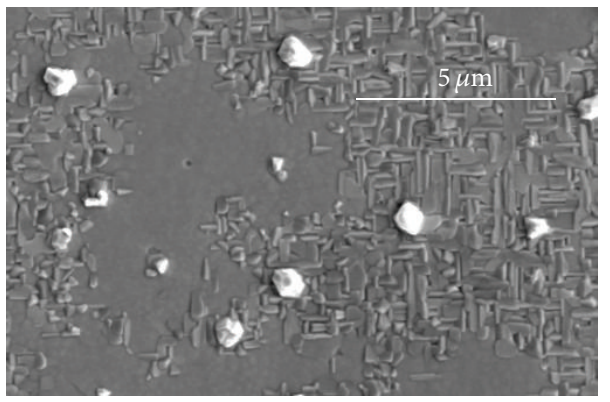

(c)

Figure 1: SEM pictures of three PCMO samples with $X \approx 0.3$ and an A-to-B site ratio close to $1(\delta \approx 0)$ on different substrates: (a) STO (sample F03_la), (b) MgO (sample E03_1), and (c) epitaxially grown Pt film on MgO (sample B03_1). The main parameters of these samples are summarized in Table 1.

In this contribution, we focus on a further important aspect of strain, that is, its effect on the appearance of crystallographic phases and solubility ranges which can induce pronounced differences to the equilibrium bulk phase diagram (e.g. [9]). Well-known examples for the change of solubility ranges are the strong increase of the Hydrogen solubility in Niobium thin film samples [10] and stressinduced precipitations of a second phase in semiconductors [11] and highly deformed metal alloys (e.g. [12]).

We show that a strain-induced precipitation of the nonequilibrium $\mathrm{MnO}_{z}$ phases takes place in ion-beam sputter (IBS) deposited PCMO thin films with nearstoichiometric composition. In contrast to film deposition by pulsed-laser deposition, where the strain strongly relaxes with increasing film thickness [4], films prepared by IBS remain in a highly strained state up to thicknesses of the order of $500 \mathrm{~nm}$. The precipitation in these films takes place over the whole nominal hole-doping range from $X=0$ to 0.5 and gives rise to the generation of growth disturbances (Figure 1). In order to investigate the influence of lattice mismatch and defect formation on the precipitation, we have used three different substrates $\left(\mathrm{SrTiO}_{3}\right.$ (STO), $\mathrm{MgO}$, and $\mathrm{Pt}$-coated $\mathrm{MgO}$ ) and two different working gases (Xe and Ar). Although the nominal mismatch of PCMO to the used substrates is tensile which would give rise to compressive outof-plane strain, the experimentally observed strain state at room temperature is quite different. Depending on the exact deposition parameters, PCMO films commonly reveal outof-plane strains in the range from $-0.3 \%$ to $-1.2 \%$ on STO, from $+0.3 \%$ to $+1 \%$ on $\mathrm{MgO}$, and from $-0.5 \%$ to $+0.4 \%$ on
Pt-coated MgO. The discrepancy between nominal lattice mismatch and strain state is due to lattice expansion by preparation induced point defects and stress relaxation which eventually poses strong bounds to the upper Mn solubility limit.

\section{Materials and Methods}

Figure 2(a) shows schematically the ion-beam sputter setup. The Kaufman source $(2.5 \mathrm{~cm}$ beam diameter, Ion Tech Inc.) generates a noble gas ( $\mathrm{Ar}$ or $\mathrm{Xe}$ ) ion beam with a small divergence which is directed onto the target. The outgoing ion beam is neutralized by an electron emitting Ar plasma source, enabling sputtering of metallic as well as dielectric compounds. In our experiments, the beam current density and the beam voltage were fixed $\left(4 \mathrm{~mA} / \mathrm{cm}^{2} / 1000 \mathrm{~V}\right)$. During deposition, the background pressure of impurity gases amounts to about $10^{-7}$ mbar. The partial pressures are $p_{\text {Ar }}=3 \times 10^{-4}$ mbar for neutralization and sputtering with Ar ions with an additional Xe inlet of $p_{\mathrm{Xe}}=1 \times 10^{-4} \mathrm{mbar}$ in the case of Xe sputtering. In order to enable nearly stoichiometric oxygen content in the films, an oxygen inlet corresponding to $p_{\mathrm{O}_{2}}=1.4 \times 10^{-4} \mathrm{mbar}$ is supplied. Unless otherwise stated, the deposition temperatures $T_{s}$ (on top of the substrate) were $T_{s}=750^{\circ} \mathrm{C}$ or $T_{s}=800^{\circ} \mathrm{C}$.

The sputter targets were prepared by a repeating milling and calcination process starting from mixture of $\mathrm{Pr}_{6} \mathrm{O}_{11}$, $\mathrm{CaCO}_{3}$, and $\mathrm{Mn}_{2} \mathrm{O}_{3}$ powders. According to $\operatorname{Pr}_{1-X} \mathrm{Ca}_{X} \mathrm{Mn}_{1+\delta}$, the composition of targets and films is characterized by the Ca content $\left(X_{\text {target }}\right.$ and $\left.X\right)$ and the Mn excess $\left(\delta_{\text {target }}\right.$ and $\left.\delta\right)$, 


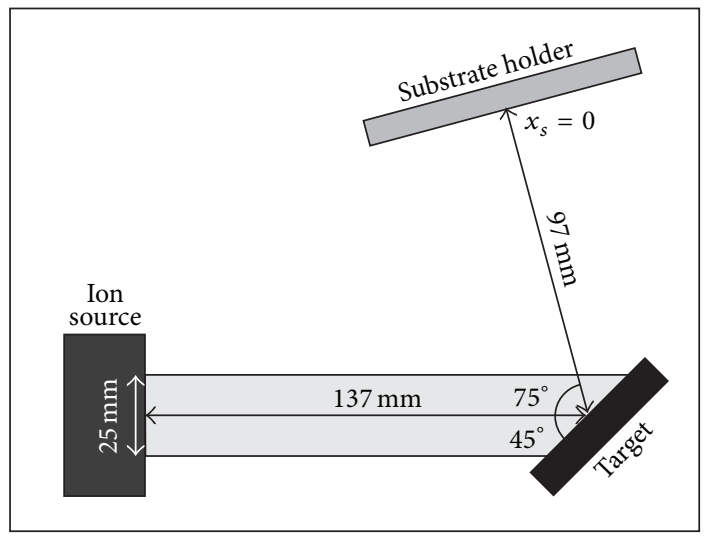

(a)

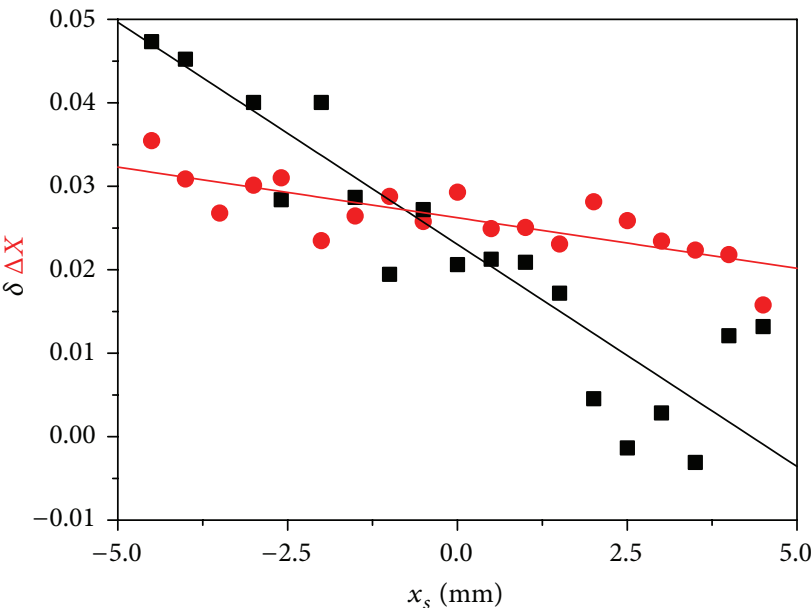

- Ca excess $\Delta X$

- Mn excess $\delta$

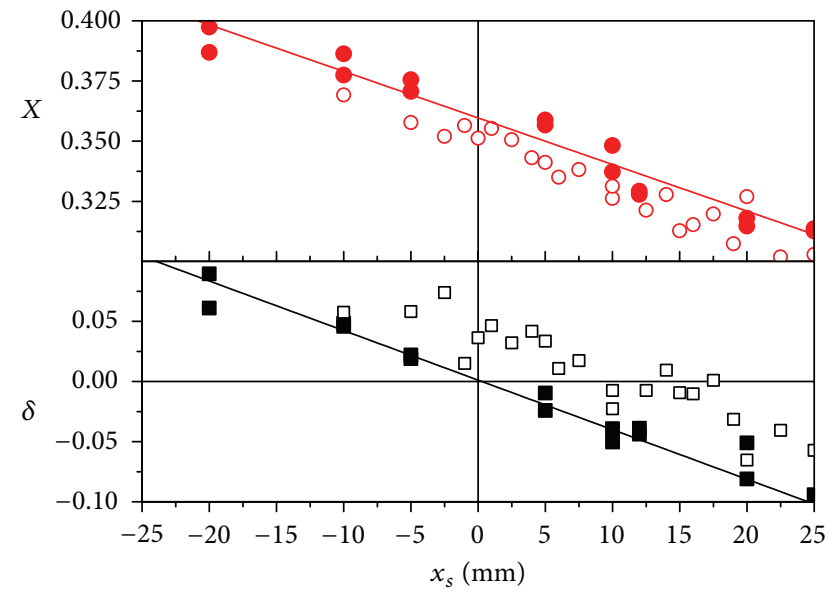

Ca content $X$ - Xe $\bigcirc \mathrm{Ar}$
Mn excess $\delta$

- Xe $\square \mathrm{Ar}$

(b)

(c)

FIGURE 2: (a) Scheme of an ion-beam sputtering device with Kaufman source, target, and substrate holder. (b) Variation of the Mn excess $(\mathrm{Pr}, \mathrm{Ca})_{1} \mathrm{Mn}_{1+\delta}$ and $\mathrm{Ca}$ excess $\Delta X=X-X_{\text {target }}$ along the substrate holder (lateral position $x_{s}$ ). The nominal composition of the target corresponds to $\delta=0$ and $X_{\text {target }}=0.28$. A $1.9 \mu \mathrm{m}$ thick film was deposited at $T_{s}=800^{\circ} \mathrm{C}$ on $\mathrm{MgO}$ in order to measure the composition by means of microprobe. (c) Variation of the Mn excess ( $\mathrm{Pr}, \mathrm{Ca})_{1} \mathrm{Mn}_{1+\delta}$ and Ca content $X$ along the substrate holder for $300 \mathrm{~nm}$ thick films prepared from the off-stoichiometric target with nominal composition $\delta=-0.02$ and $X_{\text {target }}=0.34$. Solid symbols correspond to Xe and open symbols correspond to Ar as sputter gas. The composition was measured by means of EDX including a thin film correction.

where $X$ and $\delta$ are the determined values of the thin films. We have used targets with $X_{\text {target }}=0,0.28,0.34,0.5$ and $\delta_{\text {target }}=$ 0 as well as one off-stoichiometric target with $X_{\text {target }}=0.34$ and $\delta_{\text {target }}=-0.02$. The main parameters of the film samples are summarized in Table 1. Single crystalline (100) $\mathrm{SrTiO}_{3}$ (STO) and (100) $\mathrm{MgO}$ and almost single crystalline (100) Pt films on $\mathrm{MgO}(\mathrm{Pt}-\mathrm{MgO})$ were used as substrates. The size of the substrates is $5 \times 10 \times 1 \mathrm{~mm}$. The epitaxial $300 \mathrm{~nm}$ thick Pt films were deposited via IBS at $T_{s}=750^{\circ} \mathrm{C}$.

$\mathrm{X}$-ray diffraction (XRD) studies of $\theta-2 \theta$ scans were carried out with a Bruker D8 Discover system with $\mathrm{Cu} \mathrm{K}_{\alpha}$ radiation $(\lambda=0.15418 \mathrm{~nm})$. The film composition was determined by means of Energy Dispersive X-Ray Analysis (EDX, AN 10000, Link) incorporated in a Scanning Electron Microscope (SEM, Stereoscan 360, Cambridge Instruments) and by microprobe (JEOL 8900 RL electron probe microanalyzer). For surface topology investigations, we have used a FEI Nova NanoSEM and a Nova Nano Lab 600 SEM. The latter is equipped with a Focused Ion Beam (FIB) etching device, allowing the preparation of electron-transparent lamellas for cross-sectional transmission electron microscopy (TEM) investigations. The TEM investigations were performed in a Phillips CM12 and in a FEI Titan $300 \mathrm{keV}$ equipped with an Electron Energy Loss Spectrometer (EELS) Gatan Quantum ER/965P. EDX measurement on TEM samples was done in the CM12 with a Si drift detector from Oxford with an energy resolution of $136 \mathrm{eV}$.

We have also measured the stress evolution of the PCMO films during high-temperature deposition on STO and Ptcoated $\mathrm{MgO}$ substrates by using a SIG-2000SP system from 
TABle 1: Ca content $X$, Mn excess $\delta$, substrate type, used sputter gas and thickness $d_{f}$ of the different samples, and the deposition temperature $T_{s}$. Samples with the same capital letter but different numbers were prepared in the same deposition run. Small letters indicate that samples were prepared at the same lateral position $x_{s}$ but at different vertical positions $y_{s}$. Therefore, composition and thickness of these samples are equal.

\begin{tabular}{lcccccc}
\hline Sample & $X$ & $\delta$ & Substrate & Sputter gas & $d_{f}[\mathrm{~nm}]$ & $T_{s}\left[{ }^{\circ} \mathrm{C}\right]$ \\
\hline A0_1 & 0 & 0 & STO & Xe & 410 & 675 \\
B03_1 & 0.3 & 0 & Pt/MgO & Xe & 300 & 800 \\
C03_1 & 0.35 & 0 & STNO & Xe & 110 & 800 \\
D03_1a & 0.33 & 0 & STO & $\mathrm{Ar}$ & 175 & 750 \\
D03_1b & 0.33 & 0 & $\mathrm{MgO}$ & $\mathrm{Ar}$ & 175 & 750 \\
E03_1 & 0.35 & 0 & $\mathrm{MgO}$ & $\mathrm{Xe}$ & 300 & 800 \\
F03_1a & 0.36 & 0 & $\mathrm{STO}$ & $\mathrm{Xe}$ & 130 & 750 \\
F03_1b & 0.36 & 0 & $\mathrm{MgO}$ & $\mathrm{Xe}$ & 130 & 750 \\
G03_1 & 0.35 & -0.02 & $\mathrm{STO}$ & $\mathrm{Xe}$ & 165 & 750 \\
H05_1 & 0.5 & 0 & $\mathrm{Pt} / \mathrm{MgO}$ & $\mathrm{Xe}$ & 380 & 800 \\
\hline
\end{tabular}

Sigma-Physik Messtechnik. Deposition of a stressed film on single-side clamped bar-shaped substrates $(3 \mathrm{~mm} \times 15 \mathrm{~mm}$ $\times 0.1 \mathrm{~mm}$ ) gives rise to a bending of the substrate with increasing film thickness. This bending is monitored by the change of distance between two laser spots being reflected from the substrate to the detector. The thickness dependent stress can be then calculated from Stoney's formula [13].

\section{Results}

Ion-beam sputtering takes place at a very low total pressure. Since the ejection angle of the individual target-forming elements ( $\mathrm{Pr}, \mathrm{Ca}$, and $\mathrm{Mn}$ ) is different [14] and scattering in the gas phase is almost negligible, the composition of the deposited film varies with the position along the substrate holder $x_{s}$. A typical example of this variation in composition is shown in Figure 2(b). The measurement has been performed by microprobe on a $1.9 \mu \mathrm{m}$ thick film, where no thin film corrections are required. The Ca content $X$ as well as the Mn excess $\delta$ varies almost linearly with the position on the substrate holder, but the film composition does not reach the target composition. It should be noted that the concentration does not significantly vary perpendicular to the $x_{s}$ direction; that is, in the vertical $y_{s}$ direction the homogeneity range amounts to about $15 \mathrm{~mm}$. Therefore, it is possible to prepare two films with the same composition in one run (see also caption of Table 1 ).

Figure 2(c) shows that the Ca content and the A-to-B site ratio depends on the used sputter gas, that is, $\mathrm{Xe}$ or Ar. The film compositions across the sample holder were measured by EDX on about $300 \mathrm{~nm}$ thick film sample. We have also performed a comparison between EDX and microprobe analysis. Within the scattering of the data, the slope of the concentration profiles (Ca content, Mn excess) is equal for both methods. However, most probably due to the limited energy resolution, EDX measurement reveals a slightly lower $\mathrm{Mn}$ content and a higher $\mathrm{Ca}$ content than
TABLE 2: Lattice parameters $a, b$, and $c$ for $\operatorname{Pr}_{1-X} \mathrm{Ca}_{X} \mathrm{MnO}_{3}$ (a) according to [15]. $\left\langle a_{\mathrm{pc}}\right\rangle$ is defined as the mean value of the pseudocubic lattice constant, that is, the mean value of $a / \sqrt{ } 2, b / \sqrt{ } 2$ and $c / 2$. The lattice mismatch to STO, Pt, and MgO deduced from the pseudocubic lattice constant is also included using $a_{\text {STO }}=0.3905 \mathrm{~nm}$, $a_{\mathrm{Pt}}=0.3923 \mathrm{~nm}$, and $a_{\mathrm{MgO}}=0.4213 \mathrm{~nm}$. We have also used the atom positions in the unit cell from [15] to calculate the intensity ratios by means of the CaRIne software (b).

(a)

\begin{tabular}{cccccccc}
\hline$X$ & $a[\mathrm{~nm}]$ & $b[\mathrm{~nm}]$ & $c[\mathrm{~nm}]$ & $\begin{array}{c}\left\langle a_{\mathrm{pc}}\right\rangle \\
{[\mathrm{nm}]}\end{array}$ & $\begin{array}{c}\Delta_{\mathrm{STO}} \\
{[\%]}\end{array}$ & $\begin{array}{c}\Delta_{\mathrm{Pt}} \\
{[\%]}\end{array}$ & $\begin{array}{c}\Delta_{\mathrm{MgO}} \\
{[\%]}\end{array}$ \\
\hline 0.0 & 0.5445 & 0.5809 & 0.7585 & 0.3917 & 0.30 & -0.16 & -7.03 \\
0.2 & 0.5442 & 0.5552 & 0.7657 & 0.3867 & -0.96 & -1.42 & -8.20 \\
0.3 & 0.5426 & 0.5478 & 0.7679 & 0.3850 & -1.41 & -1.86 & -8.62 \\
0.4 & 0.5415 & 0.5438 & 0.7664 & 0.3835 & -1.78 & -2.23 & -8.96 \\
0.5 & 0.5395 & 0.5430 & 0.7612 & 0.3814 & -2.34 & -2.78 & -9.48 \\
\hline
\end{tabular}

(b)

\begin{tabular}{lcc}
\hline$(h k l)$ & $d_{h k l}[\mathrm{~nm}]$ & $I[\%]$ \\
\hline$(112)$ & 0.2720 & 100.0 \\
$(200)$ & 0.2713 & 44.9 \\
$(220)$ & 0.1928 & 13.7 \\
$(004)$ & 0.1920 & 13.4 \\
\hline
\end{tabular}

microprobe analysis. In the following, we use the EDX results. Therefore, we may underestimate $\delta$ by an amount of 0.02 and overestimate $X$ by 0.028 .

PCMO films prepared by ion-beam sputtering reveal the commonly observed epitaxial relations between the deposited films and all three underlying cubic substrates. Using the Pbnm notation for PCMO, two different growth directions are observed:

$$
\text { [001] // PCMO [001] and [001] // PCMO [110] }
$$

In-plane, the $a$ and $b$ directions of (001)-grown PCMO are aligned along the $\langle 110\rangle$ directions of the substrate while the c-direction of (110)-grown domains is aligned along the $\langle 100\rangle$ and $\langle 010\rangle$ directions. Additional exchange of $\mathrm{a}$ and $\mathrm{b}$ directions gives rise to all in all six different twin domains. As a reference for samples with different $\mathrm{Ca}$ contents, we are using the Pbnm bulk lattice parameters determined by Jirak et al. [15]. These parameters and the most relevant reflection intensities are summarized in Table 2.

The Ca content, the substrate choice, and the exact deposition conditions only influence the volume fractions of the misorientations and the twin domains. PMO films $(X=$ 0 ) on STO reveal no misorientations and the dominating growth direction is $\langle 110\rangle$ for samples deposited at $T_{s} \geq$ $750^{\circ} \mathrm{C}$. A preferred [001] growth direction is only observed for lower deposition temperatures (Figure 3(a)).

Figure 3(b) shows an overview of XRD scans for two samples with a Ca content of $X=0.36$ and $\mathrm{Mn}$ excess of $\delta \approx 0$ prepared both on STO (top) and on MgO (bottom) in the same deposition run. In contrast to PMO, the two growth directions are almost indistinguishable in XRD because the 

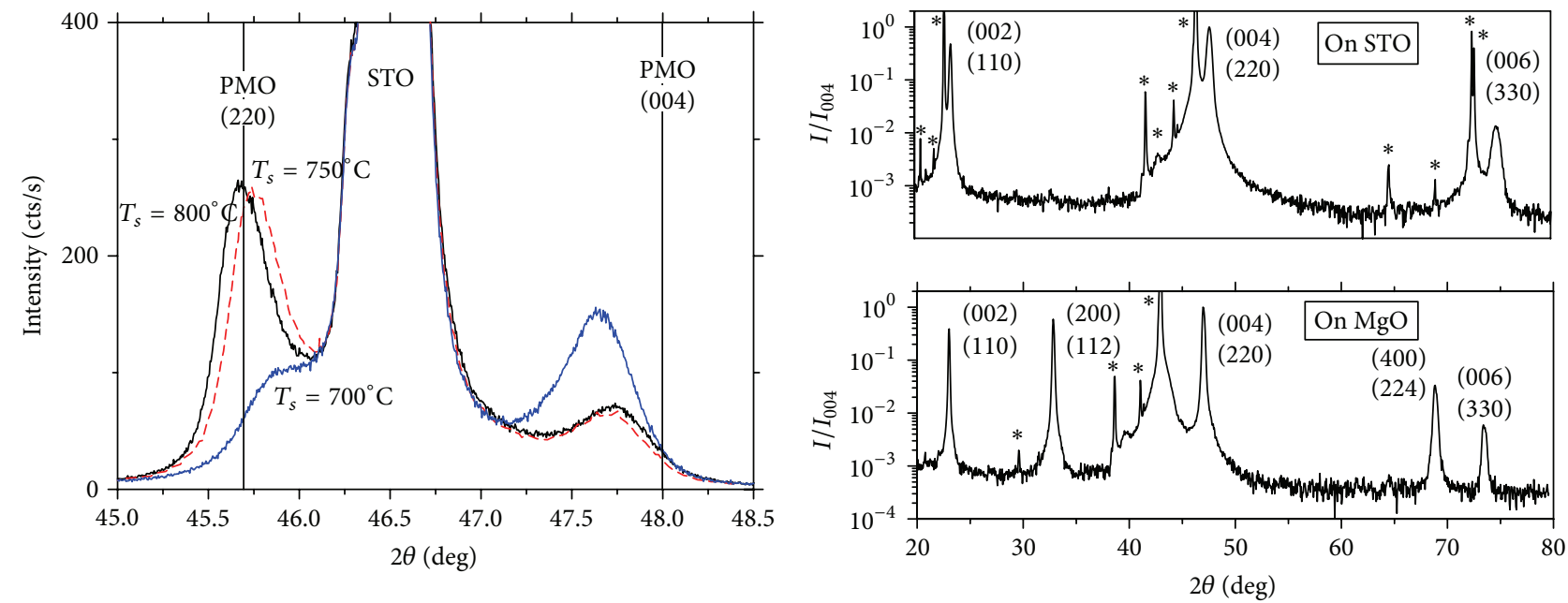

(a)

(b)

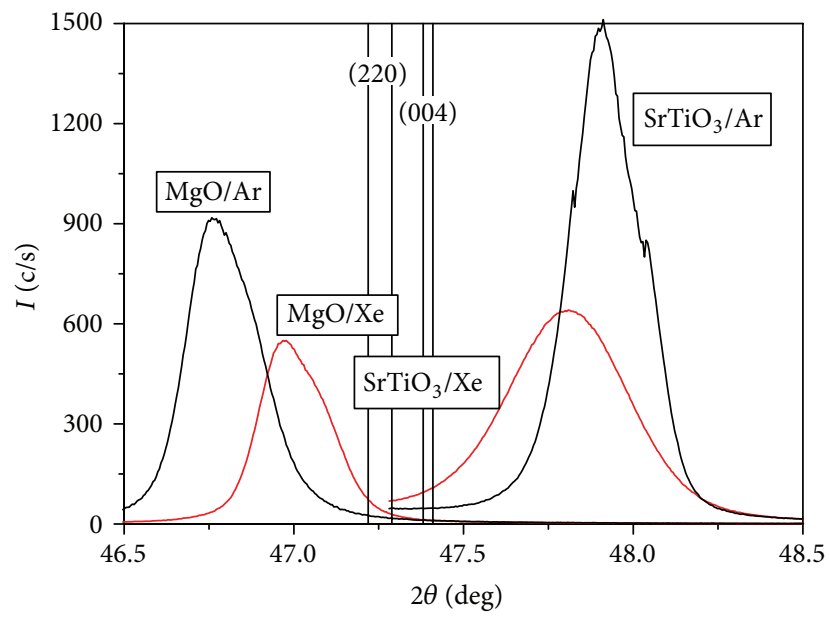

(c)

Figure 3: X-ray diffraction pattern of PCMO films. (a) Detailed scans in the vicinity of the (004)/(220) reflection of $400 \mathrm{~nm}$ thick PMO films on STO prepared by Xe sputtering at different deposition temperatures. The vertical lines have been calculated from the bulk data in Table 2 . (b) Overview: PCMO $(\delta=0, X=0.36)$ on $\mathrm{SrTiO}_{3}$ (top) and $\mathrm{MgO}$ (bottom) prepared by Xe sputtering in the same run (samples F03_la and F03_lb). The stars mark reflections which correspond to the respective substrate. This was confirmed by X-ray analysis of the noncoated substrate backsides. (c) Detailed scans in the vicinity of the (004)/(220) reflection. In addition, samples prepared by Ar sputtering $(\delta=0$ and $X=0.33$ ) are also shown (D03_la, D03_lb): the straight lines correspond to the bulk plane spacing of $d_{220}$ (left) and $d_{004}$ (right) for $X=0.33$ and 0.36 . The plane spacings have been calculated from the bulk data in Table 2.

plane spacings $d_{004}$ and $d_{220}$ are very similar. It has been pointed out that the preferable growth direction depends on the Ca content; that is, [001] growth dominates for $X=0.5$ and [110] growth dominates for $X=0.3$ [16]. However, we observe by means of TEM that films with $X \approx 0.3$ reveal large volume fraction $(\sim 75 \%)$ of the [001] growth direction.

Films prepared on $\mathrm{MgO}$ show an additional pronounced peak in XRD which corresponds to [200] or [112] growth direction. Since the structure factors of [112]/[200] are much larger than the structure factors of [220]/[004] (see Table 2), the volume fraction of this misorientation amounts only to a few volume percent for $X \approx 0.3$. This is also observed for films on $\mathrm{Pt} / \mathrm{MgO}$. In contrast, $\mathrm{PCMO}$ films with $X=0.5$ on $\mathrm{MgO}$ reveal large volume fraction of the [112] orientation, which has been also reported in [6].
Regardless of the details of composition and deposition, films prepared by ion-beam sputtering are highly strained and the strain depends on the substrate type and the used sputter gas. Figure $3(\mathrm{c})$ shows detailed scans in the vicinity of the $[220] /[004]$ reflection for samples prepared on STO and $\mathrm{MgO}$ using $\mathrm{Ar}$ and $\mathrm{Xe}$ as sputter gases. The main observations are as follows:

(i) Deposition on STO results in a decrease of the lattice spacing perpendicular to the substrate (out-of-plane) with respect to bulk lattice spacing. This implies a tensile in-plane strain state. In contrast, deposition on $\mathrm{MgO}$ causes a compressive in-plane strain state.

(ii) The asymmetry of the four reflections may indicate that the [220] volume fraction is higher on $\mathrm{MgO}$ 


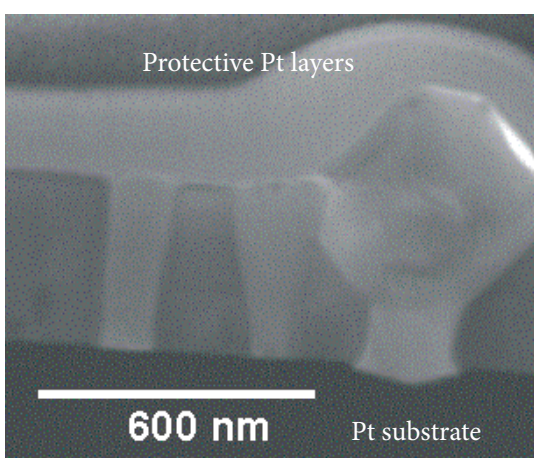

(a)

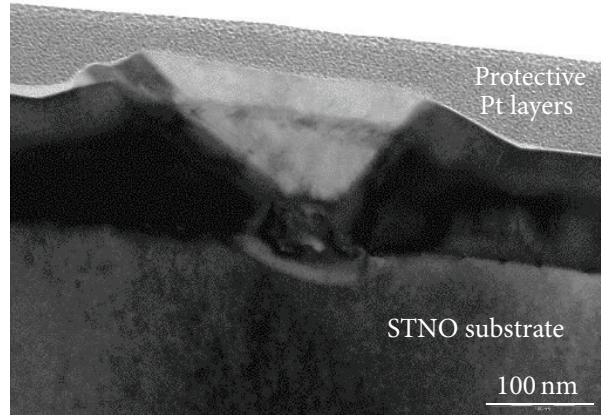

(c)
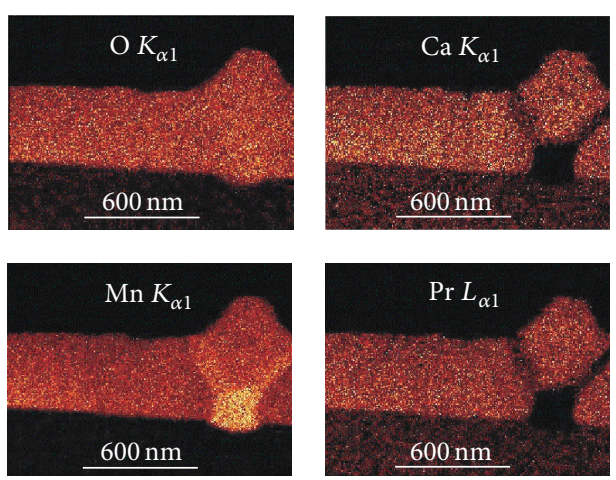

(b)

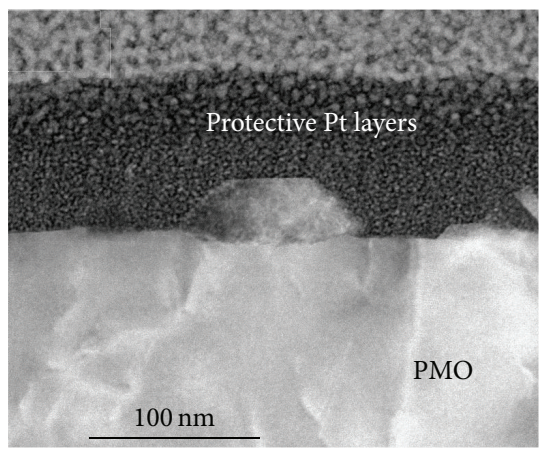

(d)

Figure 4: Cross-sectional TEM analysis of PCMO thin films $(\delta \approx 0)$. (a) PCMO $(X=0.5)$ film on Pt-coated MgO (H05_1), bright-field image of an area with two twin domains (left) and a pronounced growth disturbance (right). (b) EDX element mapping of the same area. (c) Growth disturbance in an $110 \mathrm{~nm}$ thick PCMO film $(X=0.35)$ on Nb-doped $(0.1 \mathrm{wt} . \%)$ STO substrate $\left(C 03 \_1\right)$. (d) Surface precipitate on top of a $410 \mathrm{~nm}$ thick PMO $(X=0)$ film on STO $\left(\mathrm{A} 0 \_1\right)$. The sample was postannealed for $20 \mathrm{~h}$ at $900^{\circ} \mathrm{C}$ in air.

substrates but not strongly affected by the choice of the sputter gas. Large volume fractions of this orientation have been also observed for $X=0.5$ by means of TEM.

(iii) The in-plane strain seems to be higher in case of Ar sputtering, presumably due to higher Ar incorporation. The small difference in the $\mathrm{Ca}$ content $(X=0.33$ for Ar sputtering and $X=0.36$ for $\mathrm{Xe}$ sputtering) cannot explain the large difference in lattice parameters.

In ion-beam sputtering, the emitted target atoms and, especially, the target-reflected noble gas atoms give rise to pronounced defect and strain generation [17]. This is partly compensated by defect annihilation due to the rather high deposition temperatures. The impact of reflected Ar atoms can be directly measured because of Ar-incorporation. Energy Dispersive X-Ray Analysis (EDX) of PCMO samples prepared by $\mathrm{Ar}$ as working gas reveals an Ar content of about 1 at.\%. It is interesting to note that the usage of $\mathrm{Ar}$ instead of Xe increases the compressive strain on $\mathrm{MgO}$ and the tensile strain on STO. This excludes that a simple atomic volume argument related to Ar-incorporation can explain the strain state. We rather assume that point defect generation by impinging Ar atoms is additionally influencing the substrate induced strain states.
In contrast, the $\mathrm{Xe}$ atoms with large atomic radius are hardly implemented. Xe reflection at the target only contributes to defect formation at the surface of the growing film. The full-width-half-maximums (FWHM) of the XRD reflections are larger for films prepared by means of Xe sputtering. This points to a higher degree of lattice disorder.

Independently from the Ca content and the used substrate type, PCMO films with near-stoichiometric composition $(\delta \approx 0)$ contain precipitates of a $\mathrm{MnO}_{z}$ phase which give rise to pronounced growth disturbances as shown in Figure 1. Figures 4(a) and 4(b) reveal a cross-sectional TEM bright-field image and the corresponding EDX mapping of the different elements for a PCMO film with $X=0.5$ on $\mathrm{Pt} / \mathrm{MgO}$. A precipitate nucleated at the interface between $\mathrm{Pt}$ and PCMO provokes a pronounced outgrowing PCMO misorientation. Within the resolution of the EDX system only manganese and oxygen are detected in the precipitate. The low Pr and Ca signal in the grain boundary between the PCMO outgrowth and the surrounding PCMO matrix points to a pronounced grain boundary wetting with $\mathrm{MnO}_{z}$.

Precipitation can take place at the interface to the substrate as well as in the interior (Figure 4(c)) and at the surface of PCMO (Figure $4(\mathrm{~d})$ ). We have no reliable statistic with respect to preferred nucleation sites, but it seems that all three nucleation types are present in PCMO films with $X=$ 0.3 and 0.5 . In contrast, PMO films seem to only reveal 


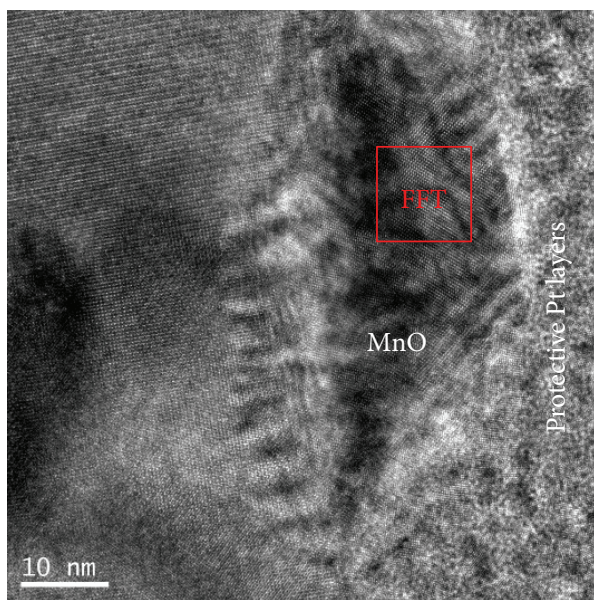

(a)

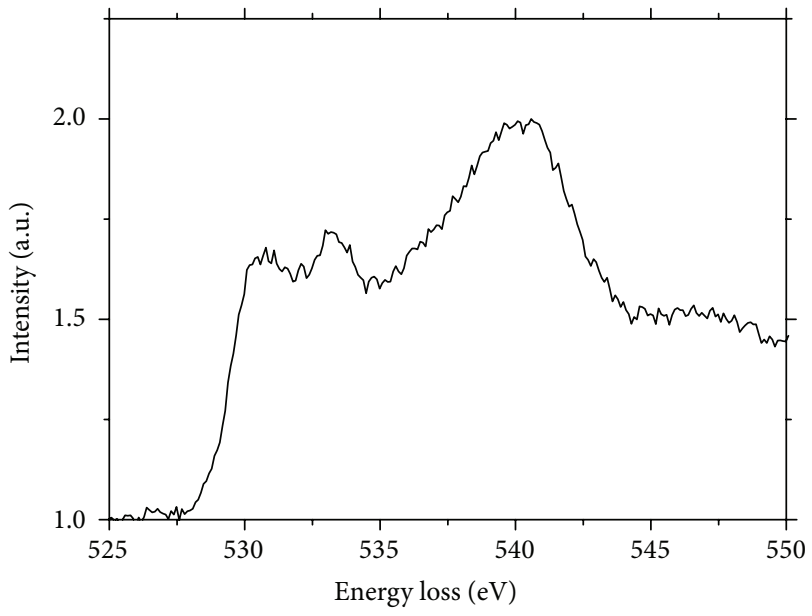

(c)

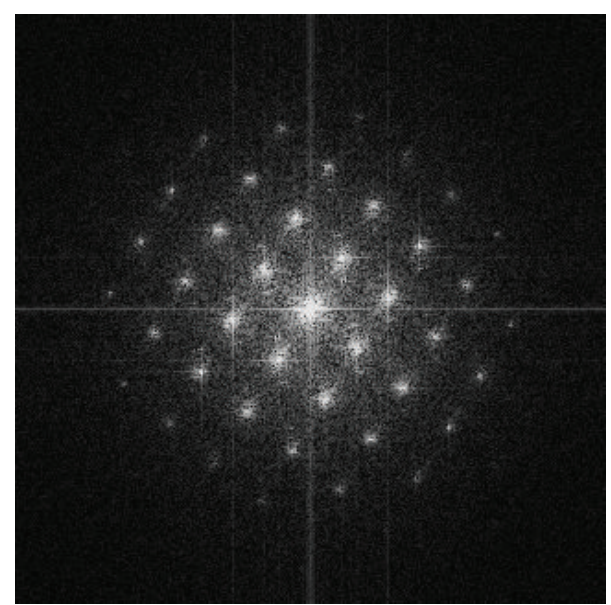

(b)

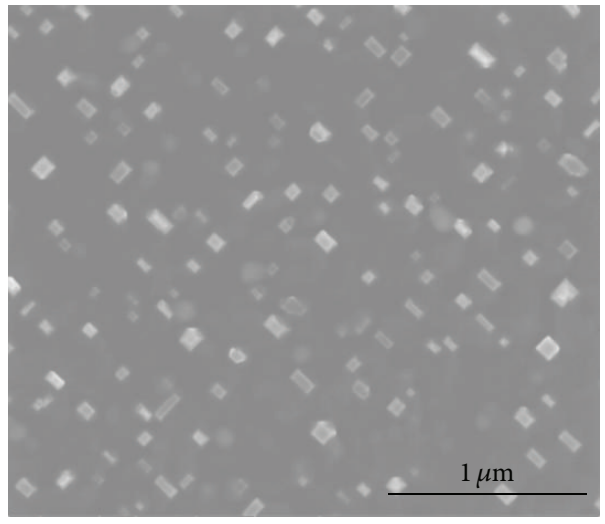

(d)

FIGURE 5: Detailed analysis of surface precipitates in PMO (sample shown in Figure 4(d)). The underlying PMO reveal two different twin domains with common [110] growth directions. (a) High-resolution image of a surface precipitates and top area of PMO. (b) FFT image of the surface precipitate. The selection for the FFT is marked in (a). (c) EELS spectrum of the oxygen K edge of a surface precipitate, which reveals the typical features of MnO [18]. (d) SEM image of the PMO surface showing very regular and well-orientated surface precipitates. The precipitate edges are parallel to the $\langle 110\rangle$ directions of the STO substrate.

surface precipitates, even in thick films. For example, the PMO film presented in Figure 4(d) is about $400 \mathrm{~nm}$ thick. Therefore, precipitation can induce different microstructures, such as embedded precipitates, surface precipitates, and near substrate precipitates leading to PCMO misorientations which protrude from the film surface.

Manganese can form different oxides, for example, $\mathrm{MnO}$, $\mathrm{Mn}_{2} \mathrm{O}_{3}, \mathrm{Mn}_{3} \mathrm{O}_{4}$, and $\mathrm{MnO}_{2}$. In order to identify the crystal structure of the $\mathrm{MnO}_{z}$ precipitates, we have analyzed the precipitates in more detail. Figure 5 shows a high-resolution image (a) and the local Fast Fourier Transformation (FFT) image of the surface precipitate (b). By taking into account the lattice parameters (see Table 3) of the different $\mathrm{MnO}_{z}$ phases, the FFT of the surface precipitate with an in-plane lattice parameter of $a_{\text {in }}=0.41 \mathrm{~nm}$ and an out-of-plane parameter of $a_{\text {oop }}=0.48 \mathrm{~nm}$ is only compatible with a highly distorted $\mathrm{MnO}$ or $\mathrm{MnO}_{2}$ phase. $\mathrm{Mn}_{2} \mathrm{O}_{3}$ and $\mathrm{Mn}_{3} \mathrm{O}_{4}$ can be excluded because the lattice parameters are too large.
TABle 3: Space group and lattice parameters $a, b$, and $c$ of the different manganese oxides.

\begin{tabular}{lccccc}
\hline & Space group & $a[\mathrm{~nm}]$ & $b[\mathrm{~nm}]$ & $c[\mathrm{~nm}]$ & Ref. \\
\hline $\mathrm{MnO}$ & $\mathrm{Fm} 3 \mathrm{~m}$ & 0.445 & 0.445 & 0.445 & {$[31]$} \\
$\mathrm{MnO}_{2}$ & $\mathrm{Pbnm}$ & 0.452 & 0.927 & 0.286 & {$[32]$} \\
$\mathrm{Mn}_{2} \mathrm{O}_{3}$ & Pcab & 0.941 & 0.942 & 0.940 & {$[33]$} \\
$\mathrm{Mn}_{3} \mathrm{O}_{4}$ & I41/amd & 0.575 & 0.575 & 0.942 & {$[34]$} \\
\hline
\end{tabular}

Comparing the EELS spectra at the oxygen $\mathrm{K}$ edge of the precipitates (Figure 5(c)) with reference data of the various manganese oxides [18] here points to the $\mathrm{MnO}$ phase.

The sample shown in Figure 5 was additionally postannealed for $20 \mathrm{~h}$ at $900^{\circ} \mathrm{C}$. From the viewpoint of the equilibrium phase diagram [19], a transition from the $\mathrm{MnO}$ phase which is only stable at low oxygen partial pressures to the $\mathrm{Mn}_{2} \mathrm{O}_{3}$ phase is expected but not observed. Consequently, 

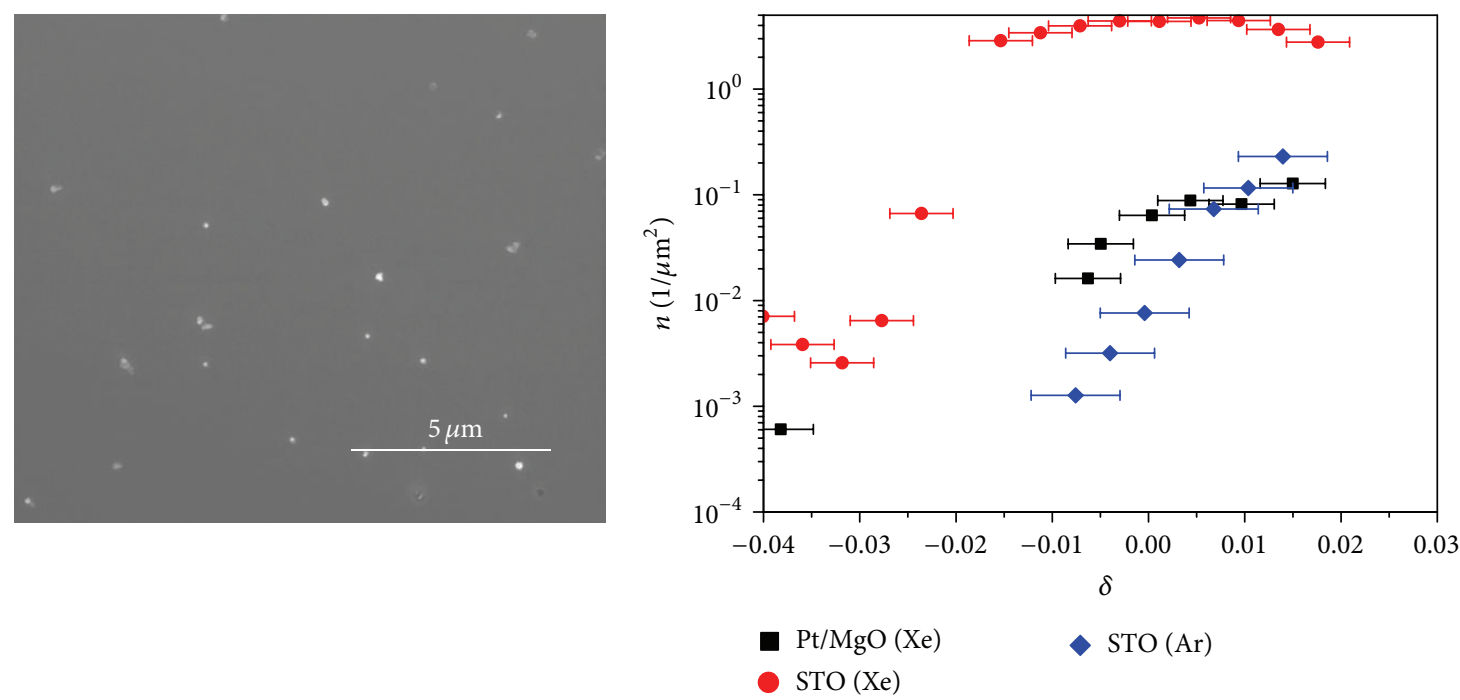

(a)

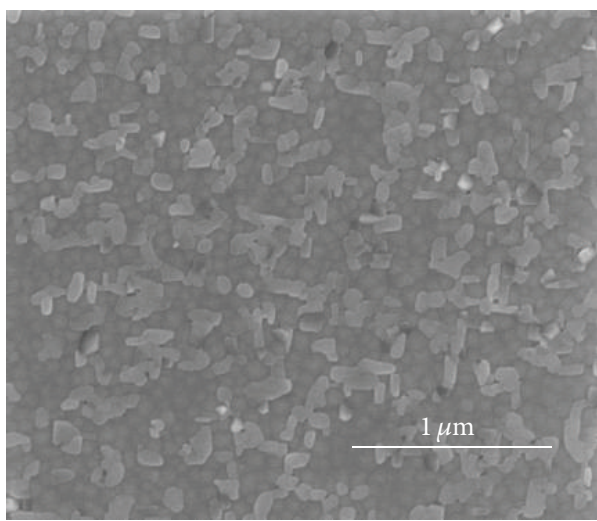

(c) (b)

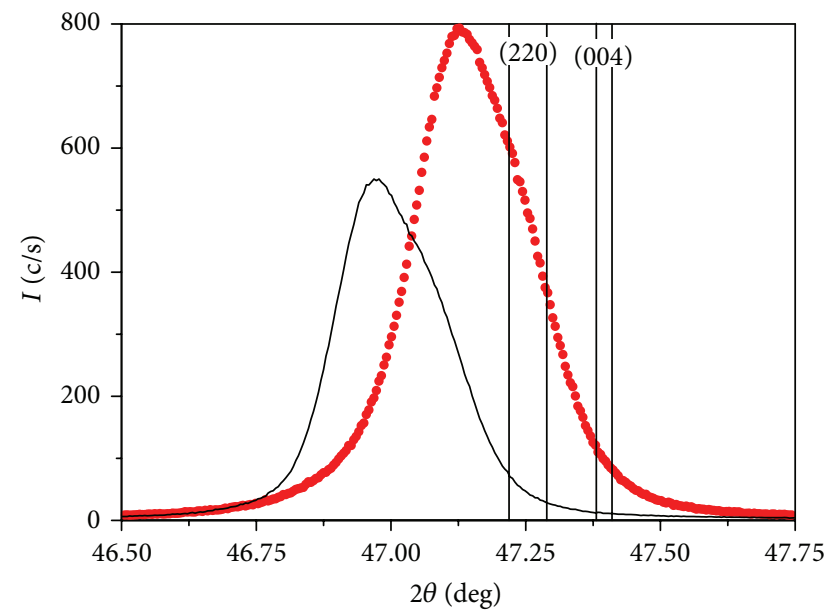

(d)

Figure 6: Outgrowth density and the influence of Mn excess for samples with $X \approx 0.3$. (a) SEM picture of a PCMO film (G03_1) with a small Mn deficit $(\delta=-0.02)$ on STO. (b) Surface density of outgrowths in dependence on the Mn excess. The Mn excess $\delta$ was deduced from EDX measurements using linear fits of the concentration profile (see also Figure 2(c)). The error bar represents the uncertainness in the concentration measurement. Same symbols belong to samples prepared in the same deposition run. The respective stoichiometric samples $(\delta=0)$ are B03_1 (black squares, substrate Pt/MgO, and sputter gas Xe), F03_la (red circles, STO, and Xe), and D03_la (blue diamonds, STO, and Ar). (c) SEM picture of a PCMO film on MgO (F03_lb). The film has the same composition $(X=0.36, \delta=0)$ and was prepared in the same run as the sample F03_la on STO shown in Figure 1(a). (d) XRD patterns of two PCMO films $(X=0.36, \delta=0)$ on MgO prepared in different runs. The sample marked by solid symbols reveals outgrowths; the second film (F03_lb, solid line) is free of outgrowths.

the $\mathrm{MnO}$ phase seems to be stabilized by coherent or semicoherent growth on the underlying PMO film. Since the $\mathrm{MnO}$ lattice constant is larger than the PMO lattice constant, such growth should give rise to compressive in-plane strain within the precipitate, that is, to the experimentally observed relation $a_{\text {in }}<a_{\text {oop }}$. The preferred orientation of the precipitate edges parallel to the [110] directions of STO also points to an epitaxial relation between $\mathrm{MnO}$ and PMO (Figure 5(d)). However, since quantitative EDX investigations on precipitates in different samples point to $\mathrm{MnO}_{2}$ and precipitation might be different in PMO and PCMO because of different misfits, we currently only would like to conclude the formation of $\mathrm{MnO}_{z}$ phases.

The TEM results imply a correlation between growth disturbances visible at the PCMO surface (Figure 1) and the presence of $\mathrm{MnO}_{z}$ precipitates. The link becomes also apparent by comparing samples prepared with different $\mathrm{Mn}$ contents. Figure 1(a) $(\delta=0)$ and Figure 6(a) $(\delta=-0.02)$ show that the surface number density of outgrowths $n$ decreases by orders of magnitude in Mn-deficit films. Plotting $n$ versus the Mn excess $\delta$ (Figure 6(b)) reveals the same trend for samples on STO and Pt-coated $\mathrm{MgO}$ substrates 
and for different sputter gases: the outgrowths vanish if the films become Mn deficient. Since $\delta$ was deduced from EDX measurements, we may slightly underestimate the Mn content by about $2 \%$. Therefore, we only state that the outgrowth vanishes close to the stoichiometric composition $\delta \approx 0$.

Depending on small change in the detailed deposition conditions, films prepared on $\mathrm{MgO}$ can reveal no $\mathrm{MnO}_{z}$ precipitates for near-stoichiometric compositions $\delta \pm 0.02$. The sample on $\mathrm{MgO}$ shown in Figure 6(c) is outgrowthfree and also cross section TEM analysis shows no $\mathrm{MnO}_{z}$ precipitates in the volume of the thin films. In contrast, the film on STO (Figure 1(a)) prepared in the same run and with the same composition (at the same position $x_{s}$ but at different positions $y_{s}$ ) reveals a high density of growth disturbances. However, the precipitation is not only controlled by the substrate choice, for example, by interfacial energies or lattice mismatch. Sometimes, PCMO films on $\mathrm{MgO}$ substrates grown under the same deposition conditions show the formation of precipitates (cf., e.g., Figures 6(c) and $1(b)$ ). It is interesting to compare samples with and without precipitates with respect to their strain state. The Xray diffraction pattern in Figure 6(d) shows that the out-ofplane strain in precipitate-free samples is significantly higher. Therefore, the precipitation is related to both the Mn content and the strain.

\section{Discussion}

As far as we know, no phase diagram of Ca-doped PMO has been published which takes Mn excess into account. The Mn solubility in the Perovskite phases of $\mathrm{LaMnO}_{3}$ and $\mathrm{PrMnO}_{3}$ corresponds to $\delta \approx 0.1$. Ca-doping of $\mathrm{LaMnO}_{3}$ seems to increase the solubility [20-22]. Such large solubilities are not observed in our PCMO thin film samples (Figure 6(b)). By taking into account the uncertainness in concentration measurement, the maximum solubility seems to be smaller than $\delta \approx 0.02$. Low deposition temperatures $T_{s}$ prohibit the formation of outgrowths. However, such films commonly reveal no CMR [23] and are of minor interest. Because of the limited statistic of TEM investigations and the uncertainness in concentration determination, we cannot quantitatively relate $\mathrm{Mn}$ excess, outgrowth density, and typical size of precipitates. However, assuming that precipitation takes place if $\delta>0$, one may write (A represents A-site cations Pr and Ca)

$$
\mathrm{A}+(1+\delta) \mathrm{Mn}+\frac{3+2 \delta}{2} \mathrm{O}_{2} \longrightarrow \mathrm{AMnO}_{3}+\delta \mathrm{MnO}_{2}
$$

Since there is a clear correlation between $\mathrm{MnO}_{z}$ formation and outgrowth density $n$, we assume that each precipitate gives rise to a visible growth disturbance. Consequently, the volume fraction of precipitates for a small excess $\delta$ is expressed as

$$
\frac{V_{\mathrm{MnO}_{2}}}{V_{\mathrm{MnO}_{2}}+V_{\mathrm{PCMO}}} \approx \delta \cdot \frac{V_{\mathrm{MnO}_{2}}^{m}}{V_{\mathrm{PCMO}}^{m}} \approx \frac{n \cdot v_{\mathrm{MnO}_{2}}}{d_{f}},
$$

where $V^{m}$ are the mole volumes, $v_{\mathrm{MnO}_{2}}$ is the mean volume of the precipitates, and $d_{f}$ is the film thickness. Using the ratio of mole volumes of about $0.46, \mathrm{Mn}$ excess of $2 \%$, and small (large) precipitates with a volume $v_{P} \approx 2.4 \times 10^{5} \mathrm{~nm}^{3}(2.0 \times$ $10^{7} \mathrm{~nm}^{3}$ ) should result in a surface density of outgrowths of $n \approx 4.2 / \mu \mathrm{m}^{2}\left(0.14 / \mu \mathrm{m}^{2}\right)$. This coarse estimations support the main trends shown in Figure 6(b): the formation of a high density of small precipitates on STO and a lower density of large precipitates with pronounced grain boundary wetting on Pt-coated $\mathrm{MgO}$ (see also Figure 4(b)).

The most probable origin of the limited solubility is the strain accompanying heteroepitaxial growth. Incorporation of Mn excess in the Perovskite phase is compensated by the formation of A-site vacancies which gives rise to a reduced unit cell volume (e.g., for $\mathrm{LaMnO}_{3}$ [20] and for $\mathrm{PrMnO}_{3}$ [24]). It has been shown by means of DFT calculations that compressive in-plane strain favors the formation of cation vacancies [25]. Although the change of the lattice constants is rather small (of the order of $-0.06 \%$ for $\mathrm{PrMnO}_{3}$ with $\delta=0.01$ [24]), a tensile lattice mismatch to the substrate may favor the formation of the stoichiometric Perovskite phase with its larger unit cell and therefore drive the precipitation of $\mathrm{MnO}_{z}$.

However, the different strain states on different substrates at room temperature cannot be directly compared without taking into account the pronounced influence of phase formation which depends on the deposition parameters. PCMO undergoes a phase transition from cubic to orthorhombic in a temperature range of $1100-1200 \mathrm{~K}$ [26] which is very close to the typical deposition temperature of about $1073 \mathrm{~K}$. The strain generated by this transition relaxes partly by twinning.

In addition to the twinning induced strain relaxation during film growth, the precipitation of $\mathrm{MnO}_{z}$ is affected by the mobility of cations. $\mathrm{MnO}_{z}$ formation requires a sufficiently high surface mobility of adatoms. Because of the pronounced wetting (Figure 4(b)), grain boundary diffusion also seems to play a significant role. It is therefore important to determine the strain evolution during deposition and cooling down to room temperature.

In order to determine the stress state during film deposition and cooling, we have performed in situ stress measurements with the hot stage. The thickness dependent stress for deposition on STO (at $T_{s}=800^{\circ} \mathrm{C}$ ) and Pt-coated $\mathrm{MgO}$ (at $T_{s}=700^{\circ} \mathrm{C}$ ) is shown in Figure 7. It is out of the frame of this contribution to discuss all details of these measurements, we will therefore mainly focus on the main observations:

(i) The stress in the early growth state of PCMO on STO is tensile and rather large (up to $4 \mathrm{GPa}$ ). Due to the large lattice mismatch $\Delta_{s}=\left(\left\langle a_{\mathrm{pc}}\right\rangle-a_{s}\right) / a_{s}$ between PCMO and STO of $\Delta_{\text {STO }}=-1.6 \%$ (see Table 1), the growth mode is most probably $3 \mathrm{D}$ growth and the island coalescence may give rise to the pronounced stress maximum at a film thickness of about $10 \mathrm{~nm}$ [27]. Although the misfit to $\mathrm{Pt}\left(\Delta_{\mathrm{Pt}}=-2.0 \%\right)$ is larger, the tensile stress is lower on Pt-coated $\mathrm{MgO}$ substrates because the elastically softer Pt film partly accommodates the misfit. Nevertheless, the stress in the early growth state is also of the order of GPa.

(ii) After the early growth stage, the stress decreases with increasing thickness but still stress remains high. The 


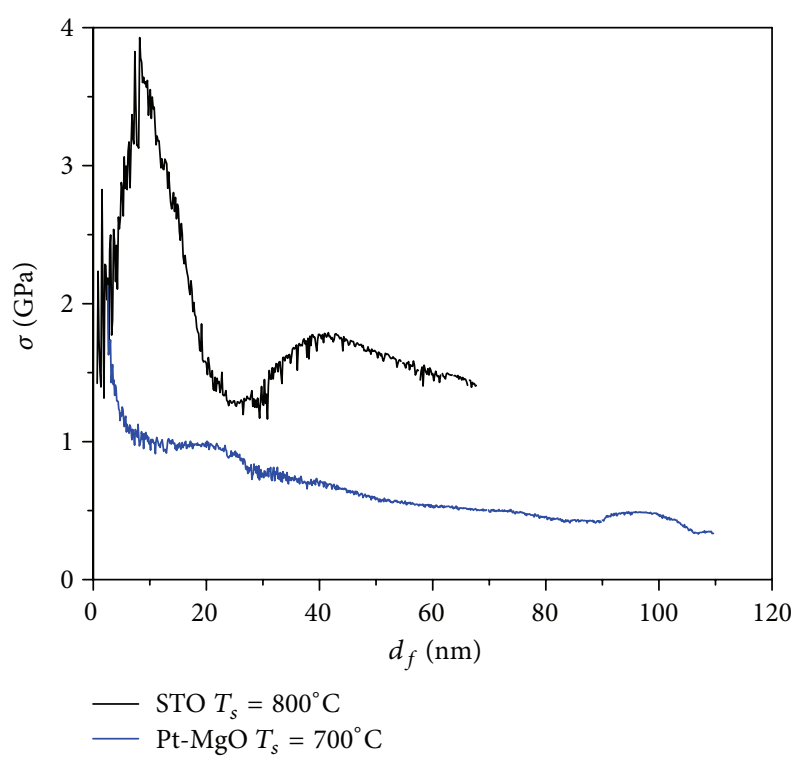

FIgURE 7: In situ determination of the thickness dependent stress during deposition of PCMO $(X=0.33, \delta=0)$ on STO and Ptcoated $\mathrm{MgO}$ substrates. The actual film thicknesses were calculated from deposition rate times time and the final thickness amounts to about $100 \mathrm{~nm}$ for both samples. In the case of the deposition on STO, the reflected laser beams moved out of the detection window above $d_{f} \approx 65 \mathrm{~nm}$. Using the linear part of the plot, we estimate the stress at $100 \mathrm{~nm}$ to be about $1 \mathrm{GPa}$. During cooling, the reflected beams moved back and the stress evolution could be measured.

almost linear decrease is not very different for samples on STO and Pt-coated MgO.

(iii) Cooling down the samples from deposition temperature to room temperature strongly modifies the stress. For the $100 \mathrm{~nm}$ thick films, the stress decreases from about $1 \mathrm{GPa}$ to $490 \mathrm{MPa}$ on STO and from $250 \mathrm{MPa}$ to $-260 \mathrm{MPa}$ in the case of Pt-coated MgO. Postmeasurement values in XRD at room temperature show that the PCMO film on STO (Pt-coated $\mathrm{MgO}$ ) reveals an out-of-plane strain of $\varepsilon_{\text {oop }}=-0.5 \%(+0.4 \%)$ which implies a tensile (compressive) in-plane strain.

It is worthwhile to note that this strong change of the stress during cooling cannot be attributed to the thermal strain induced due the difference in thermal expansion coefficient between film and substrate. Assuming for simplicity an isotropic biaxial strain state with a reasonable Poisson ratio for PCMO of $v \approx 0.35$, the stress change due to cooling from $T_{s}$ down to room temperature $T_{0}$ is given by

$$
\begin{aligned}
\frac{\Delta \sigma}{\sigma\left(T_{0}\right)} & =\frac{\sigma\left(T_{s}\right)-\sigma\left(T_{0}\right)}{\sigma\left(T_{0}\right)}=\frac{\Delta \varepsilon}{\varepsilon_{\mathrm{ip}}\left(T_{0}\right)} \\
& =-\frac{2 \cdot \nu}{1-\nu} \cdot\left(\alpha_{S}-\alpha_{\mathrm{ip}}\right) \cdot \frac{\left(T_{0}-T_{s}\right)}{\varepsilon_{\mathrm{oop}}\left(T_{0}\right)} \\
& \cong-\left(\alpha_{S}-\alpha_{\mathrm{ip}}\right) \cdot \frac{\left(T_{0}-T_{s}\right)}{\varepsilon_{\mathrm{oop}}\left(T_{0}\right)},
\end{aligned}
$$

TABLE 4: Linear thermal expansion coefficient of PCMO and substrate materials. The thermal strain $\Delta \varepsilon$ generated in PCMO films on the different substrates during cooling von $800^{\circ} \mathrm{C}$ down to room temperature is also mentioned.

\begin{tabular}{lcc}
\hline & $\alpha\left[10^{-6} / \mathrm{K}\right]$ & $\Delta \varepsilon[\%]$ \\
\hline $\mathrm{Pr}_{0.7} \mathrm{Ca}_{0.3} \mathrm{MnO}_{3}$ & $11.9[35]$ & \\
$\mathrm{SrTIO}_{3}$ & 9 & 0.23 \\
$\mathrm{Pt}$ & 8.8 & 0.24 \\
$\mathrm{MgO}$ & 8 & 0.3 \\
\hline
\end{tabular}

where $\alpha_{S}$ and $\alpha_{\text {ip }}$ are the almost constant linear thermal expansion coefficients of the substrate and the PCMO film in the high-temperature range, respectively. Since $\alpha_{\text {ip }}$ is larger than $\alpha_{S}$, only small additional tensile strain contributions are expected (see Table 4 ) in contrast to the experimental findings. For PCMO on STO, for example, the observed stress change $\Delta \sigma / \sigma \approx 1$ corresponds to the generation of an additional compressive in-plane strain which is of the order of the room temperature out-of-plane strain $\Delta \varepsilon \approx-\varepsilon_{\text {oop }}=0.5 \%$.

The defect structure of the PCMO thin films strongly affects the correlation physics of the PCMO thin films. Hoffmann et al. [23] show a systematic study of the influence of preparation induced octahedral disorder on the CMR in thin film PCMO samples with $X=0.32-0.34$ prepared by means of PLD and IBS deposition on STO and MgO. Independent of the deposition method, the CMR only can be observed if the deposition temperature is high enough (about $750^{\circ} \mathrm{C}$ ). Postannealing at temperatures above the deposition temperature increases the CMR transition temperature due to defect annihilation.

In addition to octahedral disorder, point like defects have a strong effect on the correlated electron properties. Oxygen vacancies which are formed by resistive switching can suppress the CMR effect in Pt-PCMO-Pt sandwich structures [28] and can even give rise to vacancy induced metal-insulator transitions [29]. However, the influence of point defects on the correlation properties is rather complex due to specific features of defect chemistry in manganites. For example, oxygen vacancy formation can significantly affect the A/B cation site ratio [30] with drastic effects on the ferromagnetic properties.

In the IBS PCMO films presented in this paper, Mn excess has only a minor influence, because the high stress state results in $\mathrm{MnO}_{z}$ precipitation and therefore drives the formation of a near-stoichiometric PCMO phase. Our results show that the evolving strain state and its influence on the correlation effect, for example, the CMR, are rather complex. Beside the lattice mismatch, the generation of most likely point-like defects due to the high-energy impact and the annihilation due to the high deposition temperatures has to be taken into account. In general, the flexibility of the octahedral tilt system allows for accommodation of the misfit in very thin films, which has been indeed observed for PLD films [4]. For IBS films, we observe only a moderate decrease of stress with increasing film thickness. This implies that the net defect generation rate during deposition significantly 
contributes to the stress state. This contribution does not strongly depend on the substrate choice. Defect annihilation might be the origin of the pronounced compressive stress contribution, which appears during the slow cooling down to room temperature after the deposition.

The formation of $\mathrm{MnO}_{z}$ precipitates also seems to be an important pathway of stress relaxation, because the room temperature strain of precipitate-free samples is significantly higher. Large tensile stress during deposition seems to favor the precipitation. We have not measured the stress during deposition for $\mathrm{MgO}$ substrates. Although the nominal lattice mismatch between PCMO and $\mathrm{MgO}$ is highly tensile, the room temperature strain state is compressive. We therefore expect that the misfit stress contribution to the stress-strain state of PCMO on $\mathrm{MgO}$ during deposition is not so high due to the presence of a coincidence lattice. Conclusively, the observed compressive stress must evolve because of the defect relaxation processes including twins and point defects, which are not strongly affected by the substrate choice.

Deposition at temperatures close to the cubic-orthorhombic phase transition and pronounced stress relaxation during deposition and cooling seems to be the reason for the rather broad ranges of possible strain states at room temperature on the same type of substrate. However, the appearance of precipitation is not only controlled by the lattice mismatch. Using Ar instead of Xe changes the number density of precipitates at the same Mn excess (Figure 6(b)). We suggest that the precipitation as stress relaxation takes place if the stress during deposition exceeds a critical threshold. This might explain why deposition with Xe with its high defect generation rate can result in a room temperature state with a low strain (Figure 3(b)).

\section{Conclusions}

The deposition of $\mathrm{Pr}_{1-X} \mathrm{Ca}_{X} \mathrm{MnO}_{3}$ with $X$ ranging from 0 to 0.5 by means of ion-beam sputtering is accompanied by high density of point defects which is determined by the balance of generation and annihilation rates. After termination of the film deposition, defect generation is stopped and some of the strain may be relaxed via point defect relaxation channels. However, we conclude that the formation of $\mathrm{MnO}_{z}$ precipitates represents another important relaxation path if a stress threshold is overcome. The high tensile strain during the early growth stage on STO seems to favor the formation of a high density of precipitates due to the reduction of misfit. Even if the stress is lower for the Pt-coated $\mathrm{MgO}$, the remaining stress is in the order of GPa and seems to be high enough to result in the formation of precipitates. We do not expect large tensile strain on MgO. Therefore, precipitation only takes place if the stress caused by defect formation exceeds the critical stress.

\section{Conflict of Interests}

The authors declare that there is no conflict of interests regarding the publication of this paper.

\section{Acknowledgments}

The authors gratefully acknowledge the microprobe measurements at the Geowissenschaftliches Zentrum (Georg-AugustUniversitat Goettingen, Germany) by Dr. Andreas Kronz and the financial support by the DFG within the SFB 1073 (Project B02).

\section{References}

[1] E. Dagotto, Nanoscale Phase Separation and Colossal Magnetoresistance, Springer Series in Solid-State Sciences, Springer, Berlin, Germany, 2003.

[2] J. L. García-Muñoz, J. Fontcuberta, M. Suaaidi, and X. Obradors, "Bandwidth narrowing in bulk $\mathrm{L}_{2 / 3} \mathrm{~A}_{1 / 3} \mathrm{MnO}_{3}$ magnetoresistive oxides," Journal of Physics Condensed Matter, vol. 8, no. 50, pp. L787-L793, 1996.

[3] A. J. Millis, P. B. Littlewood, and B. I. Shraiman, "Double exchange alone does not explain the resistivity of $\mathrm{La}_{1-\mathrm{x}} \mathrm{Sr}_{\mathrm{x}} \mathrm{MnO}_{3}$," Physical Review Letters, vol. 74, no. 25, pp. 5144-5147, 1995.

[4] W. Prellier, C. Simon, A. M. Haghiri-Gosnet, B. Mercey, and B. Raveau, "Thickness dependence of the stability of the chargeordered state in $\operatorname{Pr}_{0.5} \mathrm{Ca}_{0.5} \mathrm{MnO}_{3}$ thin films," Physical Review B, vol. 62, no. 24, Article ID R16337, 2000

[5] Z. Q. Yang, Y. Q. Zhang, J. Aarts, M.-Y. Wu, and H. W. Zandbergen, "Enhancing the charge ordering temperature in thin films of $\mathrm{Pr}_{0.5} \mathrm{Ca}_{0.5} \mathrm{MnO}_{3}$ by strain," Applied Physics Letters, vol. 88, no. 7, Article ID 072507, 2006.

[6] D. K. Baisnab, T. Geetha Kumary, A. T. Satya et al., "Intricacies of strain and magnetic field induced charge order melting in $\mathrm{Pr}_{0.5} \mathrm{Ca}_{0.5} \mathrm{MnO}_{3}$ thin films," Journal of Magnetism and Magnetic Materials, vol. 323, no. 22, pp. 2823-2827, 2011.

[7] T. L. Aselage, D. Emin, S. S. McCready et al., "Metal-semiconductor and magnetic transitions in compensated polycrystalline $\mathrm{La}_{1-x} \mathrm{Ca}_{x} \mathrm{MnO}_{3-\delta}(x=0.20,0.25), "$ Physical Review $B$, vol. 68 , Article ID 134448, 2003.

[8] A. Arulraj, R. Mahesh, G. N. Subbanna, R. Mahendiran, A. K. Raychadhuri, and C. N. R. Rao, "Insulator-metal transitions, giant magnetoresistance, and related aspects of the cationdeficient $\mathrm{LaMnO}_{3}$ compositions $\mathrm{La}_{1-\delta} \mathrm{MnO}_{3}$ and $\mathrm{LaMn}_{1-\delta^{\prime}} \mathrm{O}_{3}$," Journal of Solid State Chemistry, vol. 127, no. 1, pp. 87-91, 1996.

[9] A. R. Kaul, O. Y. Gorbenko, and A. A. Kamenev, "The role of heteroepitaxy in the development of new thin-film oxide-based functional materials," Russian Chemical Reviews, vol. 73, no. 9, pp. 861-880, 2004.

[10] A. Pundt and R. Kirchheim, "Hydrogen in metals: microstructural aspects," Annual Review of Materials Research, vol. 36, pp. 555-608, 2006.

[11] F. La Via, V. Privitera, C. Spinella, and E. Rimini, "Stressinduced precipitation of dopants diffused into $\mathrm{Si}$ from $\mathrm{TiSi}_{2}$ and $\mathrm{CoSi}_{2}$ implanted layers," Semiconductor Science and Technology, vol. 8, no. 7, pp. 1196-1203, 1993.

[12] S. Tian, J. Zhang, Y. Xu, Z. Hu, and H. Yang, "Stress-induced precipitation of fine gamma' -phase and thermodynamics analysis," Journal of Materials Science \& Technology, vol. 17, no. 2, pp. 257-259, 2001

[13] G. G. Stoney, "The tension of metallic films deposited by electrolysis," Proceedings of the Royal Society of London Series $A$, vol. 82, no. 553, pp. 172-175, 1909.

[14] S. D. Ekpe, L. W. Bezuidenhout, and S. K. Dew, "Deposition rate model of magnetron sputtered particles," Thin Solid Films, vol. 474, no. 1-2, pp. 330-336, 2005. 
[15] Z. Jirak, S. Krupicka, Z. Simsa, M. Dlouha, and S. Vratislav, "Neutron diffraction study of $\mathrm{P}_{1-x} \mathrm{Ca}_{x} \mathrm{MnO}_{3}$ perovskites," Journal of Magnetism and Magnetic Materials, vol. 53, p. 153, 1985.

[16] M. Fujimoto, H. Koyama, Y. Nishi et al., "Crystallographic domain structure of an epitaxial $\left(\mathrm{Pr}_{0.7} \mathrm{Ca}_{0.3}\right) \mathrm{MnO}_{3}$ thin film grown on a $\mathrm{SrTiO}_{3}$ single crystal substrate," Journal of the American Ceramic Society, vol. 90, no. 7, pp. 2205-2209, 2007.

[17] H. Windischmann, "Intrinsic stress in sputter-deposited thin films," Critical Reviews in Solid State and Materials Sciences, vol. 17, no. 6, pp. 547-596, 1992.

[18] H. Paterson, "ELNES of 3d transition-metal oxides," Ultramicroscopy, vol. 32, p. 319, 1990.

[19] R. Metselaar, R. E. J. Van Tol, and P. Piercy, "The electrical conductivity and thermoelectric power of $\mathrm{Mn}_{3} \mathrm{O}_{4}$ at high temperatures," Journal of Solid State Chemistry, vol. 38, no. 3, pp. 335-341, 1981.

[20] J. A. M. van Roosmalen, P. van Vlaanderen, E. H. P. Cordfunke, W. L. Ijdo, and D. J. W. Ijdo, "Phases in the Perovskitetype $\mathrm{LaMnO}_{3+\delta}$ solid solution and the $\mathrm{La}_{2} \mathrm{O}_{3}-\mathrm{Mn}_{2} \mathrm{O}_{3}$ phase diagram," Journal of Solid State Chemistry, vol. 114, no. 2, pp. 516-523, 1995.

[21] V. F. Balakirev, O. M. Fedorova, S. K. Estemirova, and Y. V. Golikov, "The homogeneity range of praseodymium manganite $\mathrm{Pr}_{2-x} \mathrm{Mn}_{x} \mathrm{O}_{3 \pm \delta}$ in air," Doklady Physical Chemistry, vol. 414, no. 1, pp. 110-111, 2007.

[22] F. Figueiras, J. M. Vieira, M. E. Guzik, J. Legendziewicz, P. B. Tavares, and V. S. Amaral, "The effects of $\mathrm{Ca}$ and Mn excess codoping in CMR manganites solid solution structures," Materials Science Forum, vol. 514-516, no. 1, pp. 294-298, 2006.

[23] J. Hoffmann, P. Moschkau, S. Mildner et al., "Effects of interaction and disorder on polarons in colossal resistance manganite $\operatorname{Pr}_{0.68} \mathrm{Ca}_{0.32} \mathrm{MnO}_{3}$ thin films," Materials Research Express, vol. 1 , no. 4, Article ID 046403, 2014.

[24] W. Boujelben, A. Cheikh-Rouhou, J. C. Joubert, and J. Pierre, "Stuctural and magnetic study of Perovskite manganites oxides $\mathrm{Pr}_{0.5} \mathrm{Sr}_{0.5-x} \square_{x} \mathrm{MnO}_{3}$," Physical and Chemical News, vol. 2, pp. 13, 2001.

[25] U. Aschauer, N. Vonrüti, and N. A. Spaldin, "Effect of epitaxial strain on cation and anion vacancy formation in $\mathrm{MnO}$," http://arxiv.org/abs/1506.00995vl.

[26] M. A. Carpenter, R. E. A. McKnight, C. J. Howard, and K. S. Knight, "Symmetry and strain analysis of structural phase transitions in $\operatorname{Pr}_{0.48} \mathrm{Ca}_{0.52} \mathrm{MnO}_{3}$," Physical Review $B$, vol. 82, no. 9, Article ID 094101, 2010.

[27] M. F. Doerner and W. D. Nix, "Stresses and deformation processes in thin films on substrates," Critical Reviews in Solid State and Materials Sciences, vol. 14, no. 3, pp. 225-268, 1988.

[28] M. Scherff, J. Hoffmann, B. Meyer, T. Danz, and C. Jooss, "Interplay of cross-plane polaronic transport and resistive switching in Pt- $\mathrm{Pr}_{0.67} \mathrm{Ca}_{0.33} \mathrm{MnO}_{3}-\mathrm{Pt}$ heterostructures," New Journal of Physics, vol. 15, Article ID 103008, 2013.

[29] H. S. Lee, S. G. Choi, H.-H. Park, and M. J. Rozenberg, "A new route to the Mott-Hubbard metal-insulator transition: strong correlations effects in $\mathrm{Pr}_{0.7} \mathrm{Ca}_{0.3} \mathrm{MnO}_{3}$," Scientific Reports, vol. 3, article 1704, 2013.

[30] I. Marozau, P. T. Das, M. Döbeli et al., "Influence of la and Mn vacancies on the electronic and magnetic properties of $\mathrm{LaMnO}_{3}$ thin films grown by pulsed laser deposition," Physical Review $B$, vol. 89, no. 17, Article ID 174422, 2014.

[31] B. Morosin, "Exchange striction effects in $\mathrm{MnO}$ and MnS," Physical Review B, vol. 1, no. 1, pp. 236-243, 1970.
[32] J. E. Post and P. J. Heaney, "Neutron and synchrotron X-ray diffraction study of the structures and dehydration behaviors of ramsdellite and 'groutellite," American Mineralogist, vol. 89, no. 7, pp. 969-975, 2004.

[33] S. Geller, "Structures of $\alpha-\mathrm{Mn}_{2} \mathrm{O}_{3},\left(\mathrm{Mn}_{0.983} \mathrm{Fe}_{0.017}\right)_{2} \mathrm{O}_{3}$ and $\left(\mathrm{Mn}_{0.37} \mathrm{Fe}_{0.63}\right)_{2} \mathrm{O}_{3}$ and relation to magnetic ordering," Acta Crystallographica B, vol. 27, pp. 821-828, 1971.

[34] V. Baron, J. Gutzmer, H. Rundlo, and R. Tellgren, "The influence of iron substitution on the magnetic properties of hausmannite, $\mathrm{Mn}^{2+}(\mathrm{Fe}, \mathrm{Mn})_{2}^{3+} 0_{4}$," American Mineralogist, vol. 83, no. 7-8, pp. 786-793, 1998.

[35] H. Rim, S. Jeung, E. Jung, and J. Lee, "Characteristics of $\operatorname{Pr}_{1-x} \mathrm{M}_{x} \mathrm{MnO}_{3}(\mathrm{M}=\mathrm{Ca}, \mathrm{Sr})$ as cathode material in solid oxide fuel cells," Materials Chemistry and Physics, vol. 52, no. 1, pp. 5459, 1998. 

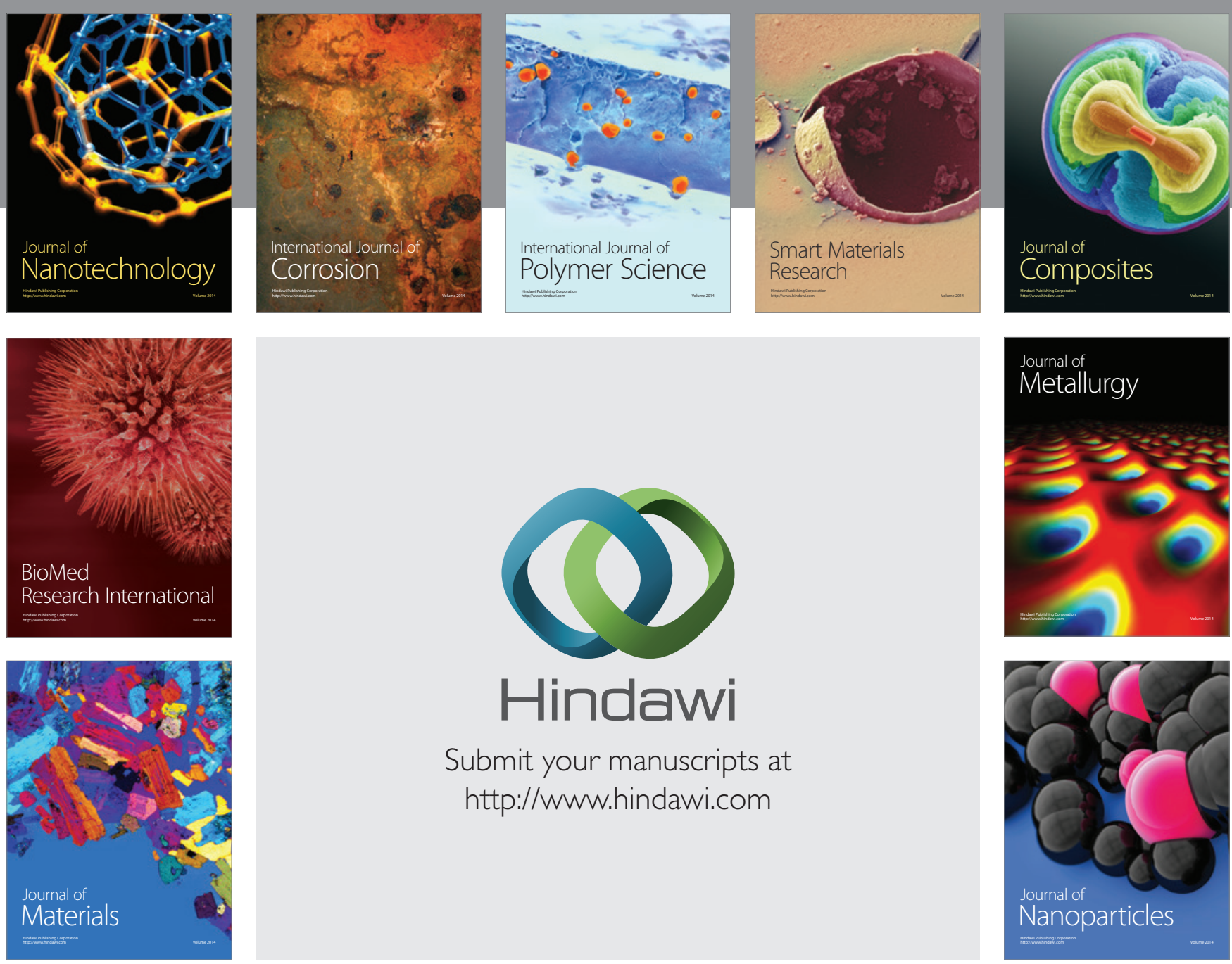

Submit your manuscripts at http://www.hindawi.com
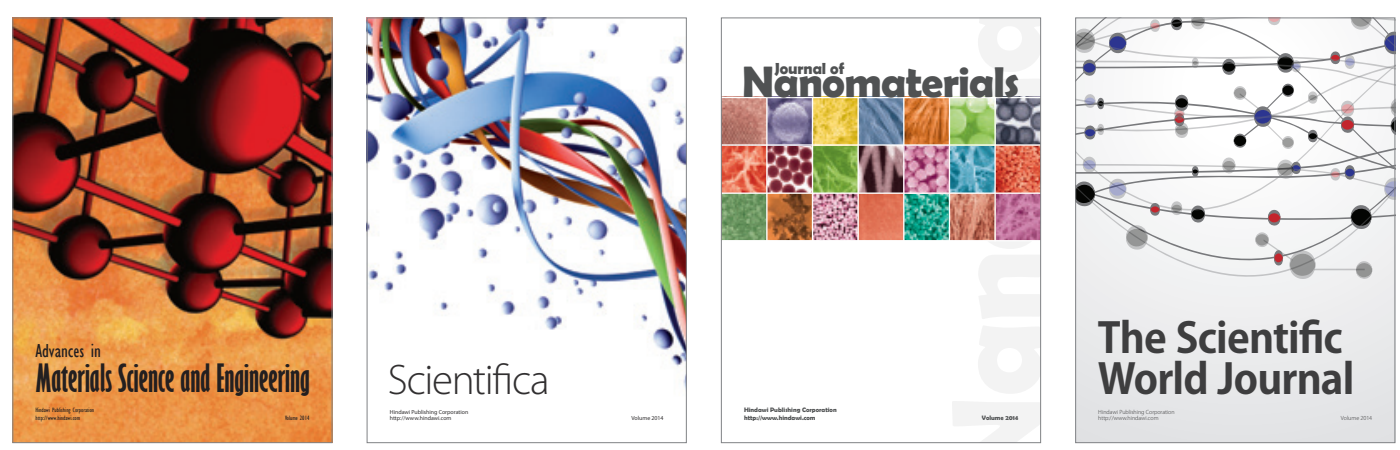

\section{The Scientific World Journal}
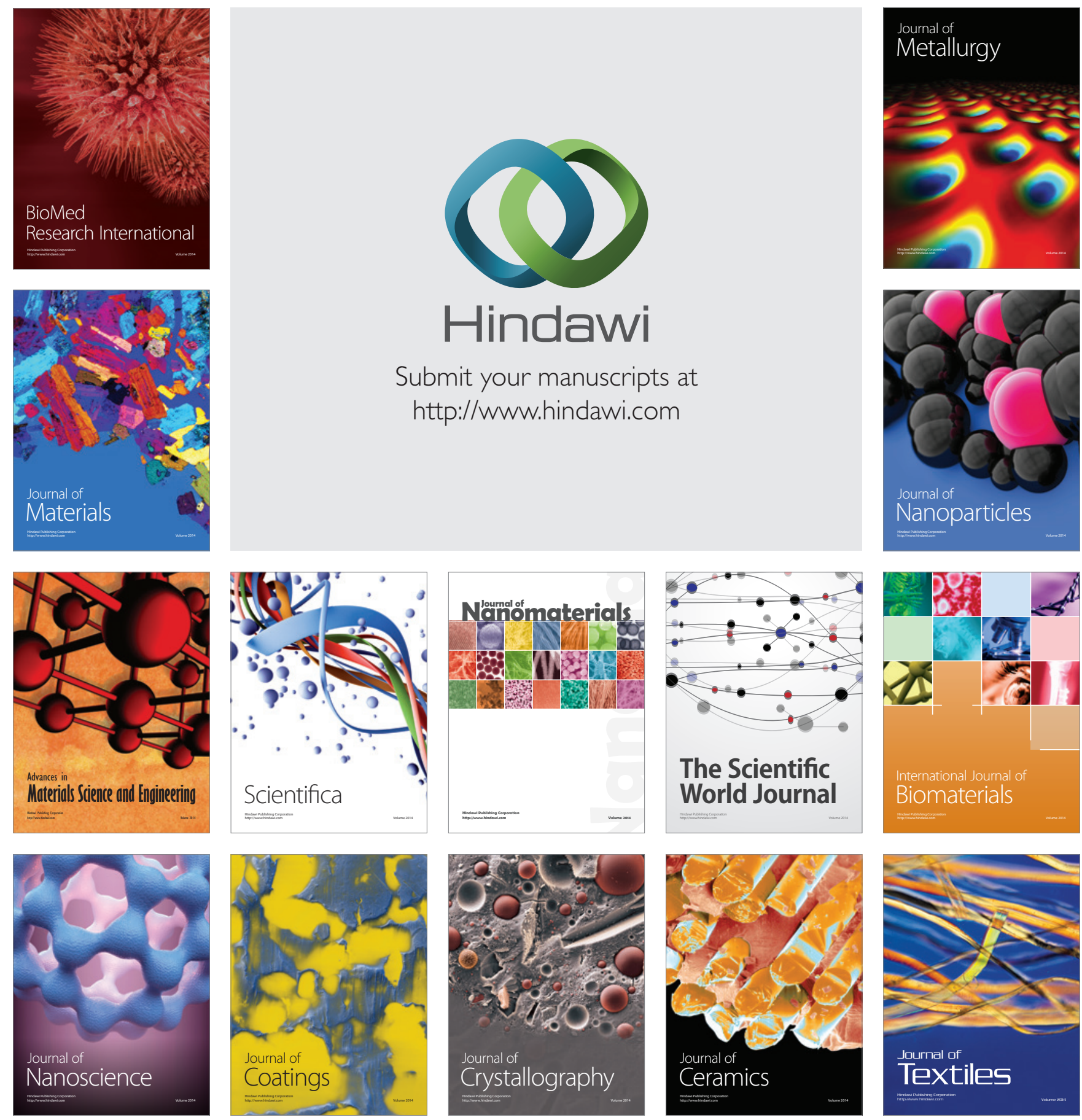